\title{
Metastatic Unresectable Sarcoma
}

National Cancer Institute

\section{Source}

National Cancer Institute. Metastatic Unresectable Sarcoma. NCI Thesaurus. Code C152074.

A metastatic sarcoma that is not amenable to surgical resection. 